\title{
Features of the structure and phase composition of materials based on aluminum oxide and chromium borides obtained under conditions of SHS and free SHS compression
}

\author{
A. P. Chizhikov ${ }^{\dagger}$, P.M. Bazhin, A. M. Stolin \\ ${ }^{\dagger}$ chij@ism.ac.ru \\ Merzhanov Institute of Structural Macrokinetics and Materials Science RAS, \\ 8 Academician Osipyan St., Chernogolovka, 142432, Russia
}

In the present work, ceramic materials based on aluminum oxide and chromium borides in the $\mathrm{Cr}_{2} \mathrm{O}_{3}-\mathrm{Al}_{-} \mathrm{B}$ system with different ratios of the starting components, from stoichiometric to excess free boron, are obtained by self-propagating high-temperature synthesis (SHS) method. Excess boron was introduced into the system in order to compensate for its loss due to burnout during SHS. It was shown that, as a result of SHS, a ceramic composite material with an $\mathrm{Al}_{2} \mathrm{O}_{3}$ matrix filled with various chromium borides: $\mathrm{CrB}, \mathrm{CrB}_{2}$, and $\mathrm{Cr}_{3} \mathrm{~B}_{4}$ is formed in the studied system. It was also shown that in the $2 \mathrm{Cr}_{2} \mathrm{O}_{3}-2 \mathrm{Al}-7 \mathrm{~B}$ system, the formation of metastable phase of aluminum borate $\mathrm{AlBO}_{3}$ is possible as a result of SHS. Since $\mathrm{AlBO}_{3}$ is a promising material for use in refractories, this system was chosen as the starting material for producing plates by the free SHS compression method. The free SHS compression method combines the synthesis of material in SHS mode with subsequent shear high-temperature deformation. The specified method allowed obtaining ceramic plates with dimensions of $50 \times 40 \times 7 \mathrm{~mm}$. According to the results of XRD and SEM, the obtained plates consisted of two phases: $\mathrm{Al}_{2} \mathrm{O}_{3}, \mathrm{CrB}_{2}$, as well as free boron. Due to changes in the conditions of heat removal under free SHS compression and a decrease in the residence time of the synthesized material at elevated temperatures, the formation of chromium monoboride and aluminum borate does not occur. Since the formation of aluminum borates begins in the temperature range $900-950^{\circ} \mathrm{C}$, an additional heat treatment of the obtained samples was carried out at a temperature of $950^{\circ} \mathrm{C}$ for 20 hours. It was found that as a result of heat treatment due to the interaction of free boron and aluminum oxide, the formation of aluminum borate $\mathrm{Al}_{4} \mathrm{~B}_{2} \mathrm{O}_{9}$ occurs, as well as the formation of chromium monoboride takes place as a result of thermal decomposition of $\mathrm{CrB}_{2}$. By this method, ceramic plates with dimensions of $50 \times 40 \times 7 \mathrm{~mm}$ were obtained. The maximum density of the obtained plates was $3.98 \mathrm{~g} / \mathrm{cm}^{3}$, while the porosity was about $15 \%$.

Keywords: self-propagating high-temperature synthesis, SHS, ceramics, deformation, alumina.

УДК: $544.45,53.091,53.092$

\section{Особенности строения и фазового состава материалов} на основе оксида алюминия и боридов хрома, полученных
в условиях СВС и свободного СВС-сжатия

Чижиков А. П. ${ }^{\dagger}$ Бажин П. М., Столин А. М.

Федеральное государственное бюджетное учреждение науки Институт структурной макрокинетики и проблем материаловедения им. А.Г. Мержанова РАН, ул. Академика Осипьяна, 8, Черноголовка, 142432, Россия

В настоящей работе методом самораспространяющегося высокотемпературного синтеза (CBC) получены керамические материалы на основе оксида алюминия и боридов хрома в системе $\mathrm{Cr}_{2} \mathrm{O}_{3}$-Al-B с различным соотношением исходных компонентов, от стехиометрического до избыточного содержания свободного бора. Избыточный бор был введен в систему с целью компенсировать его потери в результате выгорания в ходе СВС. Показано, что в результате СВС в исследуемой системе образуется керамический композиционный материал с матрицей $\mathrm{Al}_{2} \mathrm{O}_{3}$, наполненной различными боридами хрома: $\mathrm{CrB}, \mathrm{CrB}_{2}$ и $\mathrm{Cr}_{3} \mathrm{~B}_{4}$; в системе $2 \mathrm{Cr}_{2} \mathrm{O}_{3}-2 \mathrm{Al}-7 \mathrm{~B}$ в результате СВС возможно образование метастабильной фазы бората алюминия $\mathrm{AlBO}_{3}$. Поскольку $\mathrm{AlBO}_{3}$ является перспективным материалом для применения в огнеупорах, данная система выбрана в качестве исходной для получения компактных материалов методом свободного СВС-сжатия. Метод свободного СВС-сжатия сочетает в себе синтез материала в режиме 
СВС с последующим сдвиговым высокотемпературным деформированием. Согласно результатам РФА и СЭМ полученные пластины состояли из двух фаз: $\mathrm{Al}_{2} \mathrm{O}_{3}, \mathrm{CrB}_{2}$ и свободного бора. За счет изменений условий теплоотвода при свободном СВС-сжатии и снижения времени нахождения синтезированного материала при повышенной температуре образование моноборида хрома и бората алюминия не происходит. Поскольку образование боратов алюминия начинается в интервале температур $900-950^{\circ} \mathrm{C}$, то была проведена дополнительно термообработка полученных образцов при температуре $950^{\circ} \mathrm{C}$ в течении 20 часов. Установлено, что в результате термообработки за счет взаимодействия свободного бора и оксида алюминия происходит образование бората алюминия $\mathrm{Al}_{4} \mathrm{~B}_{2} \mathrm{O}_{9}$, а в результате термического разложения $\mathrm{CrB}_{2}$ возникает также моноборид хрома. Указанным методом были получены керамические пластины размерами $50 \times 40 \times 7$ мм. Максимальное значение плотности полученных пластин составило 3.98 г $/ \mathrm{cm}^{3}$, при этом пористость составила около $15 \%$.

Ключевые слова: самораспространяющийся высокотемпературный синтез, СВС, керамика, деформирование, оксид алюминия.

\section{1. Введение}

Огнеупорные материалы на сегодняшний день находят широкое применение в металлургической промышленности $[1,2]$. Они используются для футеровки высокотемпературных печей [3], металлургических конвертеров [4], ванн и ковшей для разливки металлов [5], изготовления тиглей для плавки [6] и т.д. Для промышленного производства огнеупоров чаще всего применяются материалы на основе оксидов алюминия и кремния, такие как шамот [7], каолин [8] и т.д. Однако существуют и другие виды керамических материалов, обладающих перспективными огнеупорными свойствами, например, оксид хрома $[9,10]$ и оксид циркония [11], а также муллитовая керамика [12]. Оксидные керамические материалы обладают высокой химической инертностью, что делает их перспективными для изготовления лабораторных и промышленных тиглей для расплавов и высокотемпературных испытаний [13]. В последние годы большой интерес также вызывают бораты алюминия, представляющие собой фазы, образующиеся в системе Al-B-O, благодаря своей высокой устойчивости к кислотам и щелочам, в том числе при высоких температурах, а также расплавам боратных стекол [14]. Это делает их перспективными для изготовления легковесных огнеупоров и технической керамики.

Для получения керамических материалов большой интерес представляет самораспространяющийся высокотемпературный синтез (СВС), сущность которого заключается в перемещении волны химической реакции по смеси реагентов с образованием твердых конечных продуктов [15]. При использовании СВС отсутствует необходимость во внешнем нагреве заготовки, поскольку процесс идет за счет выделяющейся в ходе химической реакции энергии. Методом СВС возможно получение широкого спектра различных материалов, в том числе оксидной керамики, муллитовой керамики, сложных керамических композиционных материалов [16,17].

Консолидация керамических огнеупорных материалов осуществляется различными методами, одним из самых распространенных является метод литья жидкого шликера $[18,19]$. Однако он обладает рядом недостатков, таких как необходимость отжига для удаления влаги, усадка в процессе сушки. Также для получения изделий из огнеупорной керамики могут применяться методы горячего прессования [20] и спекания [21], однако эти методы требуют применения интенсивного внешнего нагрева и сложного оборудования. Перспективным для получения компактных изделий на основе керамических огнеупорных материалов представляется использование сочетания процессов СВС и последующего высокотемпературного сдвигового деформирования продуктов синтеза. Такое сочетание реализовано в методе свободного СВС-сжатия, который позволяет в одну технологическую стадию получать металлокерамические пластины и слоистые композиционные материала [22,23]. Особенностью метода свободного СВС-сжатия является использование схемы деформирования с подвижными боковыми стенками. Применение такой схемы деформирования продуктов синтеза позволяет избежать пристеночного трения материала, возникающего при использовании жестких пресс-форм.

Настоящая работа посвящена исследованию особенностей структуры и фазового состава материалов на основе оксида алюминия и боридов хрома, полученных в условиях СВС без приложения внешнего усилия и в условиях свободного СВС-сжатия, который сочетает процесс СВС и высокотемпературное сдвиговое деформирование.

\section{2. Материалы и методики}

В качестве исходных для синтеза керамических материалов в настоящей работе использовались порошки $\mathrm{Cr}_{2} \mathrm{O}_{3}$ ( $\geq 99.9 \%, 1$ мкм), $\mathrm{Al}$ ( $\geq 99.5 \%$, <5 мкм) и черного аморфного бора $(\geq 99.5 \%,<10$ мкм). Указанные порошки предварительно просушивались в сушильном шкафу и смешивались в шаровой мельнице в течении 4-х часов. На основе указанных порошков готовились смеси с различным соотношением исходных компонентов, приведенных в Табл. 1.

Синтез материала в указанных системах проходил в режиме СВС за счет восстановления оксида хрома алюминием и образования боридов хрома. Адиабатическая температура горения выбранных составов приведена в Табл. 1. Расчет адиабатической температуры горения производился в программе ISMAN-Thermo.

Состав продуктов по результатам рентгенофазового анализа (РФА) рассчитывался методом корундовых чисел.

Получение компактных материалов осуществлялось методом свободного СВС-сжатия на прямоугольных заготовках массой 100 г, давление прессования составило 
Табл. 1. Характеристики исходных составов.

Table 1. Starting compounds characteristics.

\begin{tabular}{|c|c|c|c|c|c|c|c|}
\hline \multirow{2}{*}{$\begin{array}{c}\text { № состава } \\
\text { Composition } \\
\text { number }\end{array}$} & \multicolumn{3}{|c|}{$\begin{array}{c}\text { Молярное } \\
\text { соотношение } \\
\text { Molar ratio }\end{array}$} & \multicolumn{3}{|c|}{ Macc.\% / Wt.\% } & \multirow[t]{2}{*}{$\begin{array}{l}\text { Тад, }{ }^{\circ} \mathrm{C} \\
\mathrm{Tad},{ }^{\circ} \mathrm{C}\end{array}$} \\
\hline & $\mathrm{Cr}_{2} \mathrm{O}_{3}$ & $\mathrm{Al}$ & $\mathrm{B}$ & $\mathrm{Cr}_{2} \mathrm{O}_{3}$ & $\mathrm{Al}$ & B & \\
\hline 1 & 2 & 2 & 4 & 67.3 & 18.2 & 14.5 & 1776 \\
\hline 2 & 3 & 4 & 8 & 60.7 & 21.8 & 17.5 & 1807 \\
\hline 3 & 2 & 2 & 7 & 60.7 & 16.4 & 22.9 & 1995 \\
\hline
\end{tabular}

50 МПа. В результате были получены керамические пластины размерами $50 \times 40 \times 7$ мм. Плотность полученных пластин измерялась гидростатическим методом.

\section{3. Результаты и обсуждение}

Для получения материалов методом СВС без приложения внешней нагрузки в качестве объекта исследования была выбрана исходная система $\mathrm{Cr}_{2} \mathrm{O}_{3}-\mathrm{Al}-\mathrm{B}$ с различным соотношением исходных компонентов, согласно Табл. 1. Состав 1 представлял собой стехиометрическую смесь, в то время как, в составах 2 и 3 присутствует избыточное количество свободного бора. Поскольку бор имеет особенность интенсивно выгорать в процессе СВС, то было добавлено его избыточное количество с целью компенсировать выгоревшие потери. Также в системе Al-B-O характерно образование боратов алюминия при высоких температурах, начиная с $900-950^{\circ} \mathrm{C}[24,25]$. При СВС образование боратов происходит за счет взаимодействия оксида алюминия с борсодержащими соединениями. Согласно результатам РФА, приведенных в Табл. 2, в составах 1 и 2 кроме моноборида и диборида хрома, также присутствует борид хрома $\mathrm{Cr}_{3} \mathrm{~B}_{4}$. Также согласно результатам РФА в составе 3 наблюдается образование метастабильной фазы $\mathrm{AlBO}_{3}$. Образование неравновесных продуктов синтеза характерно для процессов СВС [15].

На основании результатов РФА был выбран состав 3 в качестве исходного для получения компактных керамических материалов, т.к. в нем наибольшее содержание диборида хрома, являющегося упрочняющей фазой, а также показана возможность образования бората алюминия.

При получении материалов и изделий метом свободного СВС-сжатия важным технологическим параметром является время задержки $\left(t_{3}\right)-$ время от инициирования химической реакции до приложения давления. При малых значениях данного параметра (менее 5 с) возникает ситуация, когда синтез конечных продуктов еще не завершен (даже после прохождения волны горения по образцу) и деформированию подвергаются либо смесь исходных компонентов и продуктов, либо промежуточные продукты синтеза. При завышенных значениях времени задержки (более 10 с) продукты синтеза остывают и теряют способность к пластическому деформированию. На Рис. 1 приведена зависимость плотности полученных образцов от времени задержки. Как видно из рисунка, зависимость величины плотности имеет максимум, таким образом существует оптимальный температурно-временной интервал получения изделий. Максимальное значение плотности полученных пластин составило 3.98 г/ см$^{3}$, для сравнения плотность оксида алюминия составляет $3.95 \mathrm{r} / \mathrm{cm}^{3}$.

Согласно РФА, полученные пластины состояли из двух фаз: оксида алюминия и диборида хрома, как показано на Рис. 2 а. Таким образом, на качественном уровне можно судить, что за счет изменений условий теплоотвода при свободном СВС-сжатии и снижения времени нахождения синтезированного материала при повышенной температуре, образование моноборида хрома и бората алюминия не произошло.

Изучение микроструктуры полученных компактных материалов показало, что они имеют композиционную структуру, а именно матрицу на основе оксида алюминия с распределенными в ней частицами борида хрома.

Согласно результатам энергодисперсионного анализа, приведенным на Рис. 3, светлая фаза (спектры S1, S2) соответствует бориду хрома, а темная - оксиду алюминия (спектры S3, S4). При этом по границам скоплений оксида алюминия наблюдается непрореагировавший исходный бор (спектры S5, S6). Это также подтверждается картой распределения элементов.

Поскольку в полученных материалах наблюдался свободный бор, а образование боратов алюминия начинается в интервале температур $900-950^{\circ} \mathrm{C}$, то была проведена дополнительно термообработка полученных образцов при температуре $950^{\circ} \mathrm{C}$ в течении 20 часов. Результаты РФА, полученных после термообработки материалов, приведенные на Рис. 2 b, показали наличие трех фаз: оксида алюминия, моноборида хрома и бората алюминия $\mathrm{Al}_{4} \mathrm{~B}_{2} \mathrm{O}_{9}$. Наличие моноборида хрома объяс-

Табл. 2. Фазовый состав синтезированных материалов.

Table 2. The phase composition of the synthesized materials.

\begin{tabular}{|c|c|c|c|c|c|}
\hline \multirow{2}{*}{$\begin{array}{c}\text { № состава } \\
\begin{array}{c}\text { Composition } \\
\text { number }\end{array}\end{array}$} & \multicolumn{5}{|c|}{$\begin{array}{c}\text { Продукты, масс.\% } \\
\text { Products, wt.\% }\end{array}$} \\
\cline { 2 - 7 } & $\mathrm{Al}_{2} \mathrm{O}_{3}$ & $\mathrm{CrB}_{2}$ & $\mathrm{CrB}$ & $\mathrm{Cr}_{3} \mathrm{~B}_{4}$ & $\mathrm{AlBO}_{3}$ \\
\hline 1 & 36 & 9 & 48 & 7 & - \\
\hline 2 & 64 & 25 & 8 & 3 & - \\
\hline 3 & 40 & 36 & 11 & - & 13 \\
\hline
\end{tabular}

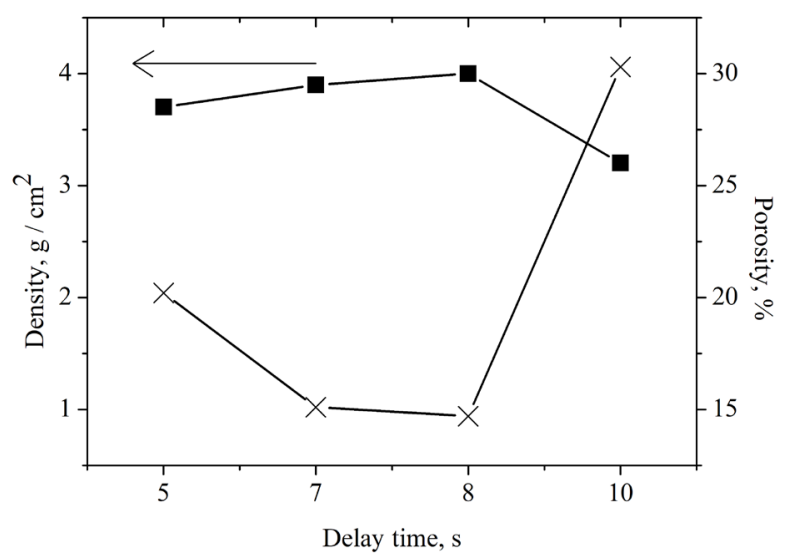

Рис. 1. Зависимость плотности полученных образцов от времени задержки.

Fig. 1. The dependence of the density of the obtained samples on the delay time. 
няется тем, что высший борид хрома при нагревании разлагается на моноборид и свободный бор.

Результаты РФА после термообработки показали, что в испытуемых образцах происходит образование бората алюминия $\mathrm{Al}_{4} \mathrm{~B}_{2} \mathrm{O}_{9}$, который, согласно диаграмме состояния $\mathrm{Al}_{2} \mathrm{O}_{3}-\mathrm{B}_{2} \mathrm{O}_{3}[26]$, является стабильным соединением, по сравнению c $\mathrm{AlBO}_{3}$. Данное соединение образуется при высокой температуре в результате взаимодействия оксида алюминия с борсодержащими соединениями, чему также способствует присутствие свободного бора в образцах. Поскольку борат алюминия обладает высокой устойчивостью к воздействию расплавов кислот, его наличие положительно влияет на эксплуатационные свойства полученных пластин.

При изучении микроструктуры образцов после термообработки можно заметить, что общий вид структуры не изменился, как показано на Рис. 4. Однако, согласно картам распределения элементов, бор в образцах больше не образует скоплений, а равномерно распределен по объему образца. Это происходит за счет образования бората алюминия в ходе термообработки.
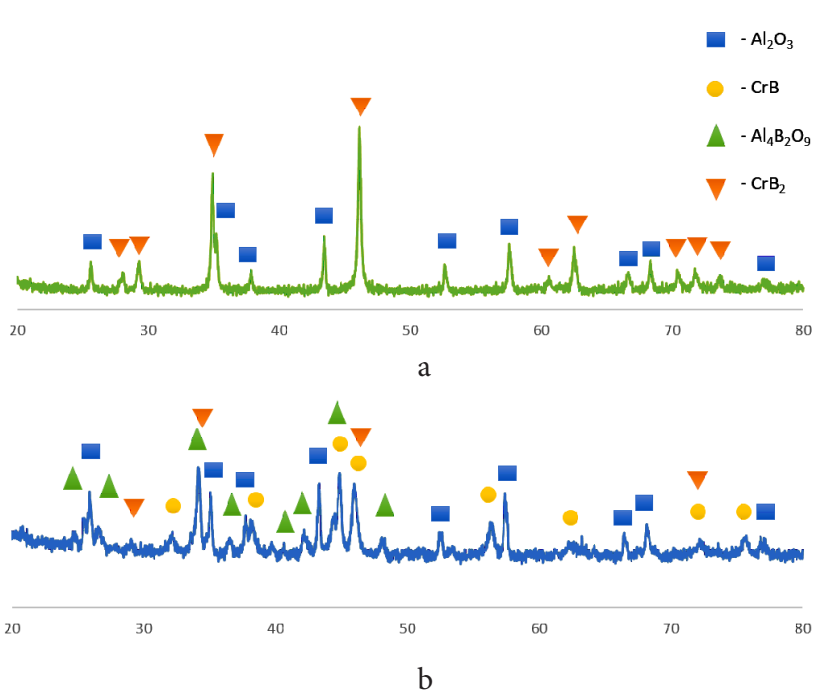

Pис. 2. (Color online) Результаты РФА полученных пластин без термообработки (a), после термообработки (b).

Fig. 2. (Color online) XRD results of the obtained plates without heat treatment (a), after heat treatment (b).

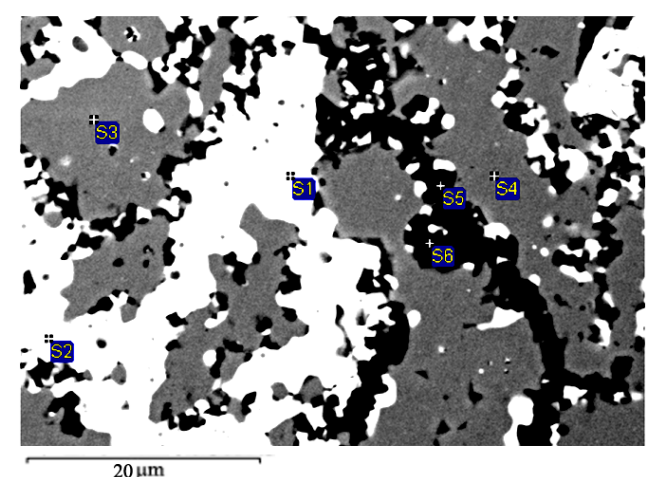

\begin{tabular}{|c|c|c|c|c|}
\hline Spectrum & B & O & Al & Cr \\
\hline S1 & 19.23 & 2.42 & 1.00 & 77.35 \\
\hline S2 & 20.68 & 2.79 & & 76.52 \\
\hline S3 & & 49.70 & 49.23 & 1.07 \\
\hline S4 & & 49.60 & 50.40 & \\
\hline S5 & 75.25 & 3.20 & 5.86 & 15.68 \\
\hline S6 & 83.77 & 2.73 & 7.14 & 6.36 \\
\hline
\end{tabular}
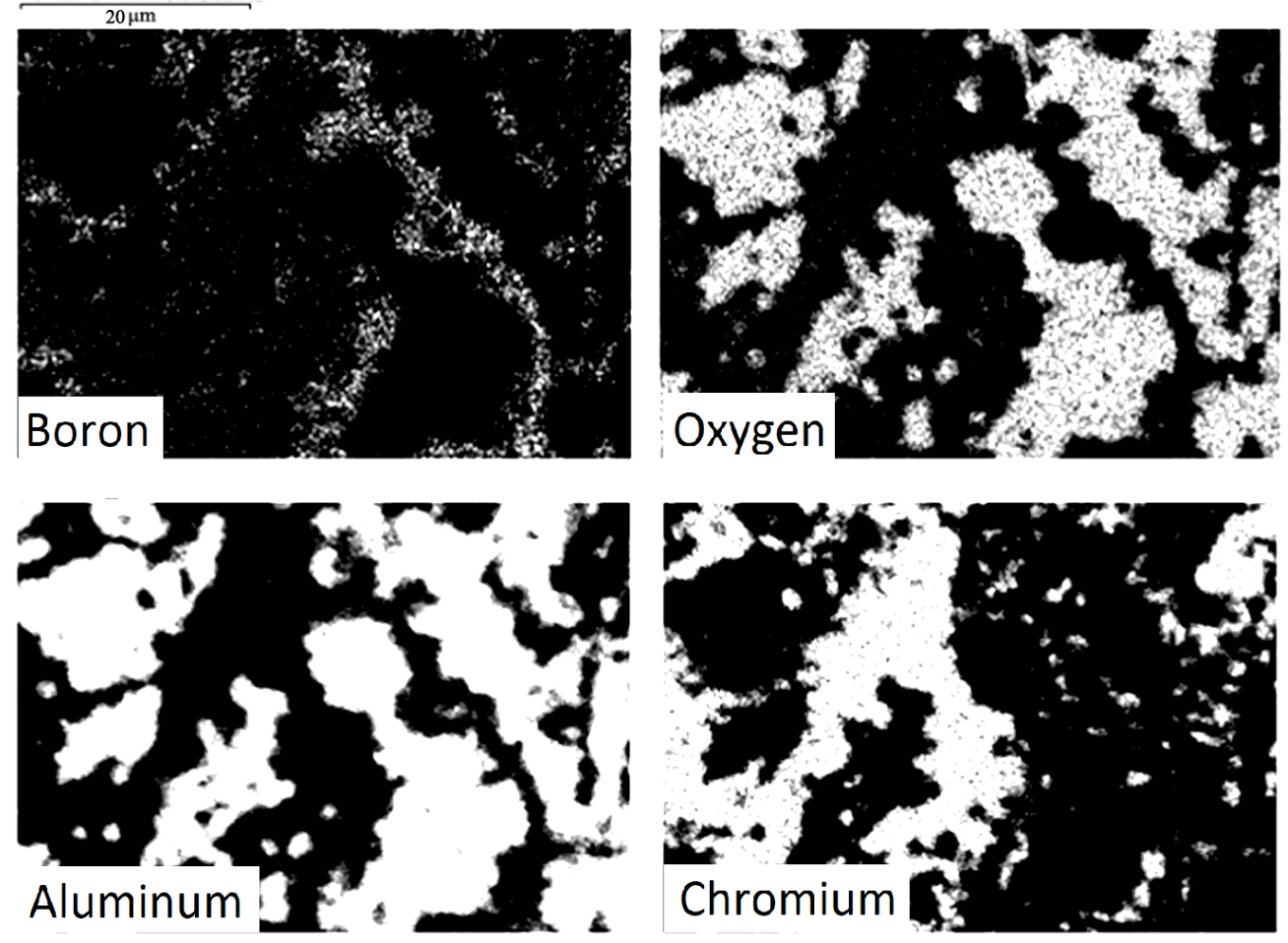

Рис. 3. Результаты ЭДС анализа и карта распределения элементов полученных пластин.

Fig. 3. The results of the EDS analysis and elements distribution map of the obtained plates. 


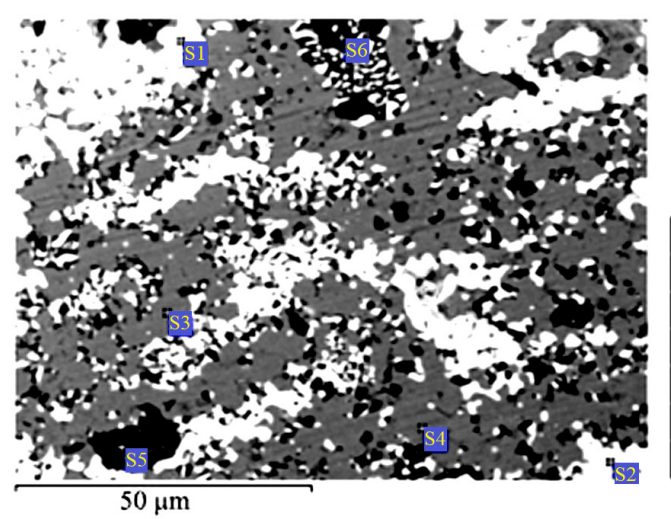

\begin{tabular}{|l|l|l|l|l|l|}
\hline Spectnum & $\mathrm{B}$ & $\mathrm{O}$ & $\mathrm{Al}$ & $\mathrm{Si}$ & $\mathrm{Cr}$ \\
\hline $\mathrm{S} 1$ & & 4.16 & & & 95.84 \\
\hline $\mathrm{S} 2$ & & 4.48 & 1.76 & & 93.76 \\
\hline $\mathrm{S} 3$ & & 52.21 & 47.79 & & \\
\hline $\mathrm{S} 4$ & & 50.66 & 47.66 & & 1.68 \\
\hline $\mathrm{S} 5$ & 40.12 & 16.80 & 24.85 & 1.75 & 16.48 \\
\hline $\mathrm{S} 6$ & 56.74 & & 20.13 & 1.97 & 21.16 \\
\hline
\end{tabular}
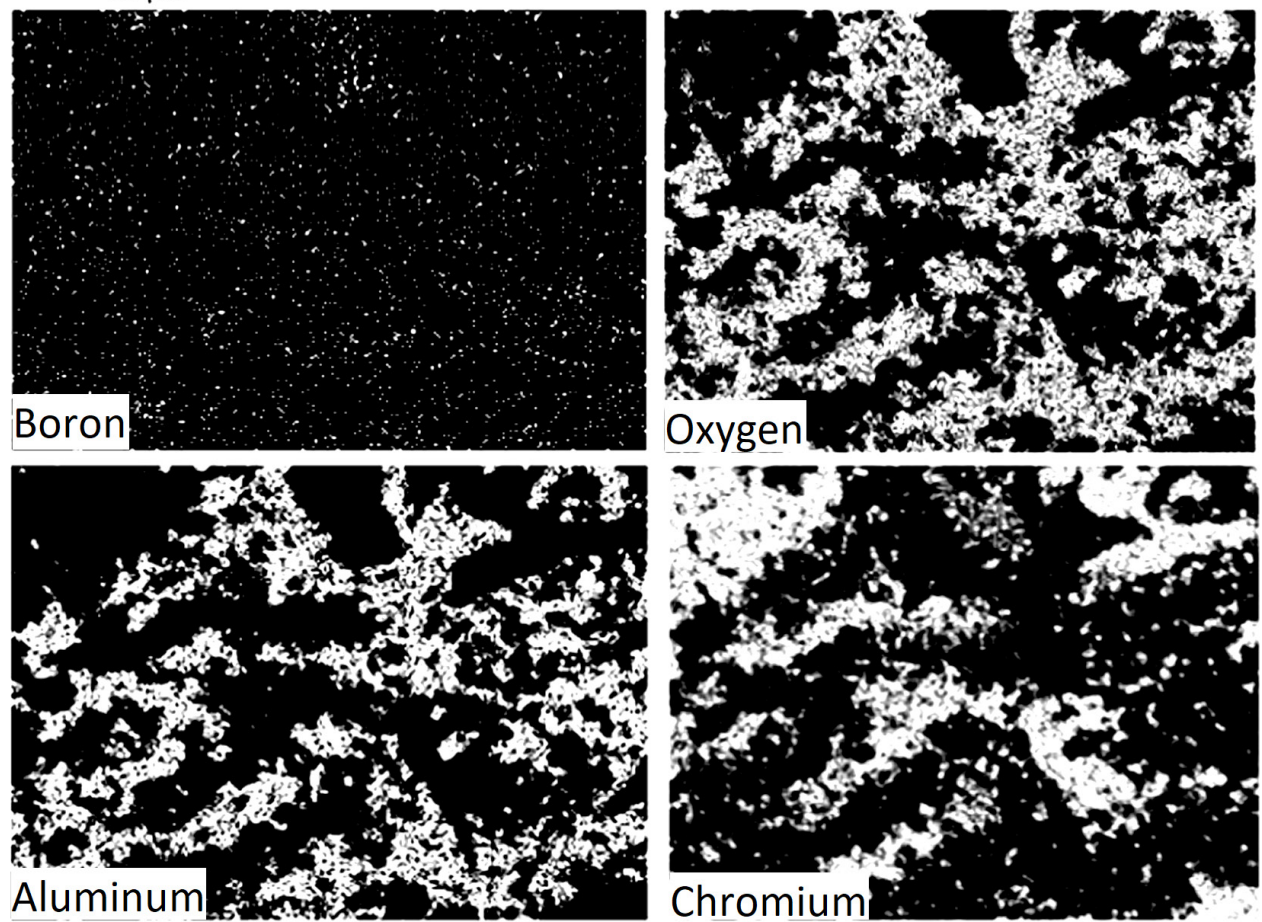

Рис. 4. Результаты ЭДС анализа и карта распределения элементов образцов после термообработки.

Fig. 4. The results of the EDS analysis and elements distribution map of the samples after heat treatment.

\section{4. Выводы}

Установлено, что содержание избыточного бора (22.9 масс.\%) в исходной смеси $\mathrm{Cr}_{2} \mathrm{O}_{3}-\mathrm{Al}-\mathrm{B}$ в условиях СВС без внешнего усилия приводит к образованию $\mathrm{Al}_{2} \mathrm{O}_{3}, \mathrm{CrB}_{2}, \mathrm{CrB}$ и метастабильной фазы бората алюминия $\mathrm{AlBO}_{3}$. Качественная оценка показала, что по сравнению с СВС при свободном СВС-сжатии за счет увеличения теплопотерь и снижения времени нахождения материала при повышенных температурах образование бората алюминия не происходит, при этом по границам оксида алюминия был расположен исходный непрореагировавший бор.

Показано, что в результате термообработки полученных в условиях свободного СВС-сжатия компактных керамических материалов, содержащих избыточный бор (22.9 масс.\%) в исходной смеси $\mathrm{Cr}_{2} \mathrm{O}_{3}-\mathrm{Al}-\mathrm{B}$, при температуре $950^{\circ} \mathrm{C}$ в течении 20 часов за счет взаимодействия свободного бора и оксида алюминия происходит образование бората алюминия $\mathrm{Al}_{4} \mathrm{~B}_{2} \mathrm{O}_{9}$, а также образование моноборида хрома в результате термического разложения $\mathrm{CrB}_{2}$.

\section{Литература/References:}

1. R. Sarkar, H. Y. Sohn. Metall Mater. Trans. B. 49 (4), 1860 (2018). Crossref

2. A. M. Abyzov. Refract. Ind. Ceram. 60 (2), 9 (2019). Crossref

3. G.Y. Wu et al. J. Alloy Compd. 796, 131 (2019). Crossref

4. V. V. Slovikovskii, A. V. Gulyaeva. Refract. Ind. Ceram. 58 (5), 475 (2018). Crossref

5. Y.L. Kats, M.V. Krasnyanskii, D.I. Yusupov, A.S. Tyuftyaev, M.K. Gadzhiev, M.A. Khromov. Metallurgist. 63 (3-4), 228 (2019). $\underline{\text { Crossref }}$

6. J.F. Chen, L.G. Chen, Y.W. Wei, N. Li, S. W. Zhang. Corros. Sci. 143, 166 (2018). Crossref

7. A.M. Hassan, H. Moselhy, M.F. Abadir. Int. J. Appl. Ceram. Tec. 16 (1), 418 (2019). Crossref

8. C. Detellier. Chem. Rec. 18 (7-8), 868 (2018). $\underline{\text { Crossref }}$

9. D.X. Pan, H.Z. Zhao, H. Zhang, P.D. Zhao, Y.C. Li, Q.F. Zou. Ceram. Int. 45 (15), 18215 (2019). Crossref

10. Y.J. Wu, S.Q. Song, Z.L. Xue, M. Nath. Metall Mater. Trans. B. 50 (2), 808 (2019). $\underline{\text { Crossref }}$ 
11. W. L. Wang, L. W. Xue, T. S. Zhang, L. J. Zhou, J. Y. Chen, Z.H. Pan. Ceram. Int. 45 (16), 20664 (2019). Crossref

12. M.A. Zalapa-Garibay, A. Arizmendi-Moraquecho, S. Y. Reyes-Lopez. J. Ceram. Sci. Technol. 10 (1), 9 (2019). Crossref

13. M.S. Varfolomeev, G.I. Shcherbakova. Refract. Ind. Ceram. 59 (3), 290 (2018). Crossref

14. M. F. Hernandez, G. Suarez, M. Cipollone, M. S. Conconi, E. F. Aglietti, N. M. Rendtorff. Ceram. Int. 43 (2), 2188 (2017). Crossref

15. E.A. Levashov, A.S. Mukasyan, A.S. Rogachev, D. V. Shtansky. Int. Mater. Rev. 62 (4), 203 (2017). Crossref

16. S. Vorotilo, A. Y. Potanin, I. V. Iatsyuk, E. A. Levashov. Adv. Eng. Mater. 20 (8), 15 (2018). Crossref

17. G. H. Liu, J. T. Li, K. X. Chen. Int. J. Refract. Met. H. 39, 90 (2013). Crossref

18. K. Moritz, N. Gerlach, J. Hubalkova, C. G. Aneziris. Int. J. Appl. Ceram. Tec. 16 (1), 14 (2019). $\underline{\text { Crossref }}$
19. S. Schaffoner, L. Freitag, J. Hubalkova, C. G. Aneziris. J. Eur. Ceram. Soc. 36 (8), 2109 (2016). Crossref

20. T.F. Krenzel, J. Schreuer, D. Laubner, M. Cichocki, H. Schneider. J.Am. Ceram. Soc. 102 (1), 416 (2019). Crossref

21. M. Nath, P. Kumar, S. Q. Song, Y.W. Li, H.S. Tripathi. Ceram. Int. 45 (9), 12411 (2019). Crossref

22. A.M. Stolin, P.M. Bazhin, A.S. Konstantinov, M. I. Alymov. Dokl. Chem. 480, 136 (2018). Crossref

23. A. Pazniak et al. Ceram. Int. 45 (2), 2020 (2019). Crossref

24. Y. Nagao, T. Hamada, A. Imamura, S. Hinokuma, Y. Nakahara, M. Machida. Catal. Sci. Technol. 6 (14), 5464 (2016). $\underline{\text { Crossref }}$

25. K. Hoffmann et al. J. Solid State Chem. 247, 173 (2017). Crossref

26. S. A. Decterov, V. Swamy, I. H. Jung. Int. J. Mater. Res. 98 (10), 987 (2007). Crossref 